\title{
The Universe itself
}

\author{
The general theory of relativity, tested time and time again, is a cornerstone of modern physics - but \\ marrying it with quantum mechanics remains a major challenge.
}

Ever since it was presented in 1915, Albert Einstein's general theory of relativity has captivated physicists and laypeople alike. Its appeal can be attributed to a variety of qualifiers, depending on one's point of view: intricacy, simplicity, genius, logic, beauty but equally so to the allure of its creator.

Einstein and his accomplishments continue to be the subject of many writings, such as John Gribbin's recent popular account of the general theory (reviewed on page 521 by Bart Verberck). Meanwhile, The Digital Einstein Papers, an open-access publishing venue collecting Einstein's scientific articles and correspondence (http://einsteinpapers.press.princeton.edu), provides a wealth of particulars on Time magazine's person of the twentieth century.

The general theory of relativity spells out how matter warps four-dimensional spacetime and how gravity arises. What is truly remarkable is that Einstein conceived it from a few basic principles such as the equivalency of inertial and gravitational mass. Indeed, there was no obvious, urgent need for the theory: Newton's theory of gravity works well enough for describing the Solar System - or for parking a satellite in an orbit around the Moon, for that matter. That being said, there was one astronomical anomaly that only Einstein's calculations correctly accounted for: the (extremely small) deviation of the precession of Mercury's perihelion from what is prescribed by Newtonian celestial mechanics.

The positive test that granted the theory enduring credibility — and Einstein instant celebrity - came in 1919. From measurements of the celestial coordinates of stars apparently near the Sun during a total solar eclipse, Arthur Eddington and his team confirmed that the Sun bends light as predicted by Einstein ${ }^{1}$.

Not everyone embraced the theory, though: in a Commentary on page 518 Milena Wazeck discusses the anti-relativist movement of the 1920s and uncovers an international network of opponents. Without any attempt at engaging in scientific argumentation, the refuters considered themselves "the last defenders of true physics". Wazeck sees parallels with adversaries of Darwinism or anthropogenic climate change.
Another prediction Einstein made is that the frequency of electromagnetic radiation changes when source and observer experience different gravitational field strengths - gravitational redshift. This effect was confirmed in 1959 by Robert Pound and Glen Rebka, who performed an experiment where the redshift of photons travelling along a lift shaft was counterbalanced by the Doppler shift of the moving detector ${ }^{2}$.

Arguably one of the most famous checks of the theory was carried out in 1971 by Joseph Hafele and Richard Keating, who took atomic clocks on round-the-world flights. Owing to height variations, the clocks accumulated nanosecond time differences agreeing with those expected from the special and general theories of relativity combined ${ }^{3,4}$.

Gravitational waves, another of Einstein's predictions, are often poetically described as ripples in the fabric of spacetime. Believed to result from violent cosmic events, direct evidence has yet to be obtained. The first results from the Advanced Laser Interferometer Gravitational Wave Observatory, a large terrestrial facility where reflected laser beams are scrutinized for signs of interactions with gravitational waves, are expected later this year, which will also see the launch of LISA Pathfinder, a spacecraft loaded with instruments for the detection of spacetime ripples through freefall experiments in outer space.

Black holes are probably the most popular by-products of the general theory, and it is easy to understand why they have entered the public domain - the idea of a region of spacetime from which nothing, at least classically, can escape, does indeed fire the imagination. Since the discovery of the first pulsar (a rotating neutron star) in 1967, black holes are believed to really exist - a black hole is formed when a neutron star collapses. Pulsars themselves also played a major role in the general theory's success story: the orbiting pattern of a binary pulsar (a system of two stars, one of which is a pulsar) exhibits a 'periastron shift' analogous to Mercury's perihelion shift. Radio spectra of binary pulsars reflect this effect.

With all the high-precision testing of relativity, and all the excitement about astrophysical phenomena it explains or predicts, one should not forget the profound consequences the general theory has on our understanding of the dynamics of the Universe itself.

Einstein was puzzled at first that his equations allow solutions implying an expanding or a contracting universe. But further theoretical elaborations by others, together with Edwin Hubble's discovery of galaxies speeding away from us wherever we look, firmly established the notion of the Big Bang and the expanding Universe, ingredients that form essential parts of today's standard model of cosmology.

As impressive as the general theory of relativity undoubtedly is, it is uncomfortably separated from quantum mechanics. The oddities of curved spacetime, dictating gravity and the cosmos at large, don't really have a detectable impact on the largely microscopic quantum world. However, attempts to bring the two formalisms together are ongoing 5 .

From an experimental point of view, studying the interface between quantum physics and gravity is extremely challenging - whatever the observable effect to measure, it would be tiny. Nevertheless, progress in the design of experiments sensitive enough for establishing a 'quantum gravity phenomenology', with a focus on laboratory-bound tests as an alternative to astrophysical observations, is being made ${ }^{6}$.

Much purely theoretical work is usually focused on finding an answer to what happens at the Planck scale: masses, lengths or times where the strength of gravity becomes comparable to the other fundamental interactions (which are currently unified in the standard model of particle physics). One difficulty is that the general theory cannot be reformulated as a quantum field theory; another problem is that the inherent indeterminacies of quantum mechanics become dominant. Many approaches are being pursued, of which string theory is probably the best known.

One hundred years on, one could say that the general theory of relativity still refuses to come to terms with quantum physics - in imitation of its maker.

\footnotetext{
References

1. Dyson, F. W., Eddington, A. S. \& Davidson, C. Phil. Trans. R. Soc. A 220, 291-333 (1920).

2. Pound, R. V. \& Rebka, G. A. Phys. Rev. Lett. 3, 439-441 (1959).

3. Hafele, J. C. \& Keating, R. E. Science 177, 166-168 (1972)

4. Hafele, J. C. \& Keating, R. E. Science 177, 168-170 (1972)

5. Amelino-Camelia, G. Living. Rev. Rel. 16, 5 (2013).

6. Amelino-Camelia, G. Nature Phys. 10, 254-255 (2014).
} 